\title{
In Vitro Replication of Adeno-Associated Virus DNA
}

\author{
TIE-HUA NI, XIAOHUAI ZHOU, DOUGLAS M. MCCARTY, IRENE ZOLOTUKHIN, \\ AND NICHOLAS MUZYCZKA* \\ Department of Microbiology, State University of New York at Stony Brook Medical School, \\ Stony Brook, New York 11794
}

Received 27 July 1993/Accepted 12 November 1993

\begin{abstract}
The study of eukaryotic viral DNA replication in vitro has led to the identification of cellular enzymes involved in DNA replication. Adeno-associated virus (AAV) is distinct from previously reported systems in that it is believed to replicate entirely by leading-strand DNA synthesis and requires coinfection with adenovirus to establish completely permissive replication. In previous work, we demonstrated that two of the AAV nonstructural proteins, Rep78 and -68, are site-specific endonucleases and DNA helicases that are capable of resolving covalently closed AAV termini, a key step in AAV DNA replication. We have now cloned the AAV nonstructural proteins Rep78, Rep68, and Rep52 in the baculovirus expression system. Using the baculovirusexpressed proteins, we have developed an efficient in vitro AAV DNA replication system which mimics the in vivo behavior of AAV in every respect. With no-end AAV DNA as the starting substrate, the reaction required an adenovirus-infected cell extract and the presence of either Rep78 or Rep68. Rep52, as expected, did not support DNA replication. A mutant in the AAV terminal resolution site (trs) was defective for DNA replication in the in vitro assay. Little, if any, product was formed in the absence of the adenovirus-infected HeLa cell extract. In general, uninfected HeLa extracts were less efficient in supporting AAV DNA replication than adenovirus-infected extracts. Thus, the requirement for adenovirus infection in vivo was partially duplicated in vitro. The reduced ability of uninfected HeLa extracts to support complete DNA replication was not due to a defect in terminal resolution but rather to a defect in the reinitiation reaction or in elongation. Rep78 produced a characteristic monomer-dimer pattern of replicative intermediates, but surprisingly, Rep68 produced little, if any, dimer replicative form. The reaction had a significant lag $(30 \mathrm{~min})$ before incorporation of ${ }^{32}$ P-deoxynucleoside triphosphate could be detected in DpnI-resistant monomer replicative form and was linear for at least $4 \mathrm{~h}$ after the lag. The rate of incorporation in the reaction was comparable to that in the simian virus 40 in vitro system. Replication of the complete AAV DNA molecule was demonstrated by the following criteria. (i) Most of the monomer and dimer product DNAs were completely resistant to digestion with DpnI. (ii) Virtually all of the starting substrate was converted to heavy-light or heavy-heavy product DNA in the presence of bromo-dUTP when examined on $\mathrm{CsCl}$ density gradients. (iii) Both flip and flop orientations of the terminal repeat were present in the product DNA, suggesting that each of the terminal repeats underwent at least two rounds of terminal resolution. The availability of an efficient in vitro AAV DNA replication assay should help to identify cellular proteins required exclusively for leading-strand DNA synthesis.
\end{abstract}

Much of what is known about eukaryotic DNA replication has come from in vitro studies of the replication of simian virus 40 (SV40), a small DNA virus which relies largely on cellular replication enzymes for amplification $(5,18,20,21,30,33,49$, $50,52-56$ ). Adeno-associated virus (AAV) is a single-stranded human DNA virus whose genome size is approximately $5 \mathrm{~kb}$ (43; see references 1 and 31 for reviews). Like SV40, AAV appears to rely on cellular enzymes for DNA replication $(1,3$, 17, 31, 45). However, unlike SV40, AAV appears to use exclusively leading-strand DNA synthesis (Fig. 1) (2, 9, 10, 24, $25,46,47)$. Thus, the development of an efficient in vitro AAV DNA replication system may be useful for identifying cellular enzymes that are used primarily for leading-strand synthesis. In addition, AAV is unusual in that coinfection with a helper virus (either herpesvirus or adenovirus [Ad]) is essential for establishing a completely permissive environment for viral amplification $(1,31)$. In the case of Ad, which has been studied extensively, it appears that the helper virus is used primarily for the induction of AAV or cellular genes that are required for

\footnotetext{
* Corresponding author. Mailing address: Department of Microbiology, State University of New York at Stony Brook, Life Sciences Building 260, Stony Brook, NY 11794-5222. Phone: (516) 632-8817. Fax: (516) 632-8891.
}

AAV replication $(1,3,17,31,45)$. The Ad DNA polymerase and terminal protein are not required for AAV DNA synthesis, and the Ad DNA-binding protein, although serving an essential helper function, appears to be required primarily for efficient AAV gene expression $(3,17,45)$.

Two AAV genes are required for viral DNA replication. The first is the viral origin of DNA replication $(9,25,37,39)$, which consists of a 145-bp terminal repeat (TR). The second is the rep gene, which codes for four nonstructural proteins that are synthesized from a single open reading frame by the use of alternate promoters and splicing $(1,31,43)$. Viruses with mutations in the two larger proteins, Rep78 and Rep68, are defective for viral DNA replication $(11,48)$, while mutations in the smaller Rep proteins, Rep52 and Rep40, do not affect the production of replicative-form (RF) DNA (4). Like other parvoviruses, the AAV genome replicates by a self-priming strand displacement mechanism $(2,9,10,24,25,35,46,47)$. The first step in DNA replication is the conversion of the single-stranded input genome into a linear duplex molecule in which at least one of the ends of the molecule is covalently joined. This is accomplished by using the terminal AAV palindrome as the primer for synthesis of the complementary strand. The covalently joined end is then converted into an open duplex end by a process called terminal resolution or hairpin tiansfer (Fig. 1) $(2,42,47)$. A consequence of terminal 


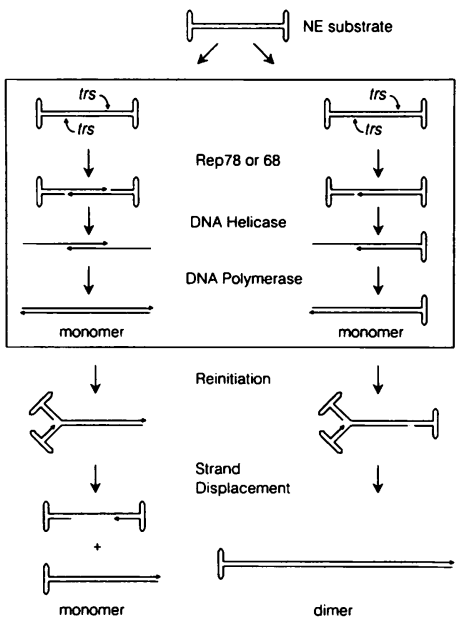

FIG. 1. Mechanism of AAV DNA replication. The diagram illustrates the key steps during AAV DNA replication that are expected to occur when NE DNA is used as the starting substrate. The left-hand series of reactions shows how monomer duplexes are generated, and the right-hand series illustrates how a dimer molecule might be generated. The box highlights the steps during terminal resolution. Horizontal arrows indicate $3^{\prime}$ ends, and trs indicates the terminal resolution site. The nick at the trs site is catalyzed by either Rep78 or Rep68 $(15,16)$. Although the Rep proteins are known to have DNA helicase activity $(15,16)$, the proteins responsible for the remaining enzymatic steps have not been identified unequivocally. See the text for additional details.

resolution is that the terminal AAV sequence is inverted (24, $25,35,42$ ). Following resolution, the ends of the molecule are believed to reform the terminal hairpins, thus providing a $3^{\prime}$ $\mathrm{OH}$ primer for strand displacement synthesis (reinitiation [Fig. 1]). Elongation from the hairpin primer then generates a single-stranded genome (which is presumably packaged) and a new RF molecule which again can undergo terminal resolution. Several other types of RF molecules can theoretically be formed during AAV replication. One of these is a dimer RF which presumably is formed when strand displacement synthesis proceeds through a terminal sequence that has not yet been resolved (Fig. 1). The two major forms of RF species found in vivo are monomer and dimer duplex molecules (46).

In previous work, we have purified Rep68 to apparent homogeneity from AAV-infected cells and have partially purified $\operatorname{Rep} 78$ and $\operatorname{Rep} 52(15,16)$. In addition, we developed several in vitro assays to characterize the biochemical activities of these proteins. For a substrate, we used a linear AAV DNA molecule in which both ends were covalently joined (Fig. 1), called no-end (NE) DNA (42). We demonstrated that both Rep78 and Rep68 were capable of correctly resolving a hairpinned end when supplemented with an uninfected HeLa cell extract $(15,16,40,42)$ to provide DNA polymerase activity. In the absence of a HeLa cell extract, both Rep78 and Rep68 could bind to the AAV terminal hairpin and cut at the terminal resolution site (trs). The trs endonuclease reaction was ATP dependent and was both site specific and strand specific, resulting in a singly nicked product that contained a molecule of Rep covalently attached at the trs site $(15,16,40)$. Finally, both enzymes were found to have an ATP-dependent DNA helicase activity $(15,16)$. In contrast, Rep52 had neither terminal resolution nor trs endonuclease activity, and we could detect no specific binding to the hairpin $(14,16)$.

Although these experiments provided a plausible, direct role for the two larger Rep proteins in DNA replication, they were not successful in producing net DNA synthesis in vitro. Two other laboratories have reported the replication of AAV DNA in vitro. Yalkinoglu et al. (59) reported that a circular plasmid containing a single AAV terminal repeat replicated as a circle in an in vitro assay supplemented with crude extracts from Chinese hamster or mouse cells. DNA synthesis in this system was dependent on the presence of an AAV TR and was stimulated by pretreatment of the cells with a carcinogen. This was consistent with in vivo studies which showed that transformed cells treated with carcinogens became partially permissive for AAV replication $(38,57,58)$. However, the absence of a requirement for viral Rep proteins and the production of circular rather than linear intermediates suggested that this system did not accurately reflect the conditions of fully permissive AAV replication. Hong et al. (12) have also described an in vitro replication system in which the substrate was a circular plasmid containing a complete copy of AAV. This assay produced linear replicative intermediates but was relatively inefficient, producing only a limited amount of replication over an extended period of time. In this article, we describe the development of an efficient in vitro AAV DNA replication assay using NE DNA as the substrate. The reaction mimics the in vivo behavior of AAV DNA replication in virtually every respect.

\section{MATERIALS AND METHODS}

Cells, plasmids, and virus. Cells, plasmids, and virus stocks were prepared and maintained as previously described $(16,29)$. Mammalian cells were routinely tested for latent AAV proviruses as described elsewhere (29). SF9 insect cells, wild-type baculovirus, and the baculovirus transfer plasmids described below were obtained from M. Summers (Texas A\&M University) and maintained as described elsewhere $(22,23)$.

Substrates and chromatography materials. NE substrate DNA was prepared from psub201 plasmid DNA as previously described $(36,42)$. Ribo- and deoxyribonucleoside triphosphates were purchased from Pharmacia or Sigma. They were dissolved in water and neutralized with $\mathrm{NaOH}$. Radioactive nucleotides were purchased from ICN. Creatine phosphate, creatine phosphokinase, and dithiothreitol (DTT) were purchased from Sigma and dissolved in water. Restriction and DNA modifying enzymes and $\lambda$ bacteriophage DNA were from New England Biolabs. Molecular weight protein standards were from Bio-Rad. DEAE cellulose and phosphocellulose were purchased from Whatman; mono-S and mono-Q fast protein liquid chromatography columns were purchased from Pharmacia.

Baculovirus expression vectors. AAV Rep52 was expressed from a recombinant baculovirus containing AAV nucleotides 964 to 4493 which constituted the $B c l I-t o-X b a I$ fragment from the previously described plasmid pIM29 (28). The plasmid used to generate this recombinant was derived from pNM348, which contained the $X b a I$ fragment of pIM29 in the polylinker $X b a I$ site of the baculovirus transfer vector pAC610 $(22,26)$. pNM348 was subsequently grown in Escherichia coli dam $\mathrm{dcm}$, and the $B c l I-t o-X b a I$ fragment was recloned into BamHI- and $X b a$ I-digested pAC610 to generate a baculovirus transfer construct in which the first ATG start codon under the control of the polyhedron promoter initiates translation of the p19 rep gene product. This plasmid, $348 d \mathrm{Bcl}$ (Fig. 2), was cotransfected with purified wild-type Autographa californica nuclear polyhedrosis virus DNA into SF9 insect cells, and occlusionnegative recombinant virus plaques were isolated. Expression 

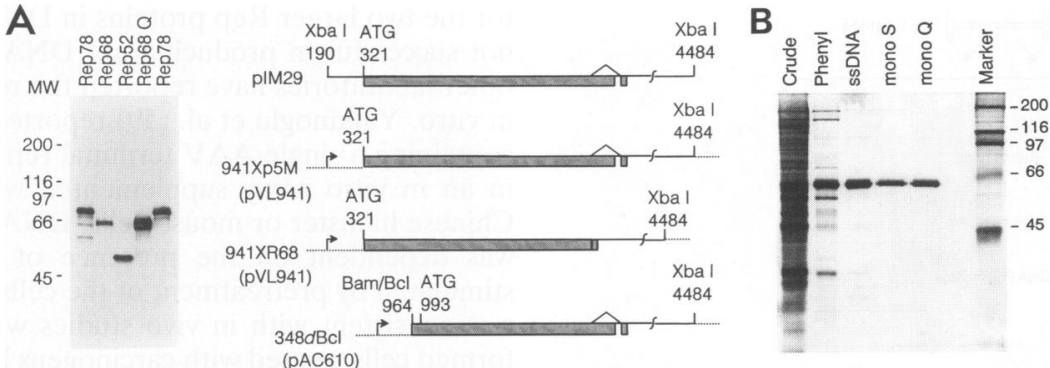

FIG. 2. Baculovirus-expressed Rep proteins. (A) Aliquots (6.8 $\mu \mathrm{g}$ each) of $1 \mathrm{M}$ nuclear salt extracts of baculovirus-infected cells expressing Rep78, Rep68, or Rep52 were electrophoresed on an SDS-10\% polyacrylamide gel and transferred to a nitrocellulose filter. Two different preparations of Rep78 (the two outside lanes) were loaded on the gel. In addition, $0.03 \mu \mathrm{g}$ of the baculovirus Rep68 mono-Q fraction was loaded (lane Rep68 Q). The filter was incubated sequentially with anti-52/40 Rep mouse monoclonal antibody (13) and anti-mouse horseradish peroxidase-coupled antibody. Protein molecular mass markers were rabbit myosin $(200 \mathrm{kDa})$, E. coli $\beta$-galactosidase $(116 \mathrm{kDa})$, rabbit muscle phosphorylase B $(97 \mathrm{kDa})$, bovine serum albumin $(66 \mathrm{kDa})$, and chicken ovalbumin $(45 \mathrm{kDa})$. The diagram indicates the AAV sequences from the parental plasmid pIM29 that were cloned into the baculovirus transfer plasmids (pVL941 and pAC610) to make the three Rep transfer plasmids used for cloning Rep into baculovirus under polyhedron promoter control. See Materials and Methods for additional details of the construction. (B) Purification of baculovirus-expressed Rep68. Aliquots of the different steps in the purification of Rep68 were fractionated by electrophoresis on an SDS-10\% polyacrylamide gel and then stained with silver. The following amounts of each fraction were loaded: $1 \mathrm{M}$ nuclear extract (Crude), $6.2 \mu \mathrm{g}$; phenysepharose (Phenyl) fraction, $1 \mu \mathrm{g}$; single-stranded (ss) DNA cellulose fraction, $1.8 \mu \mathrm{g}$; mono-S fraction, $1.1 \mu \mathrm{g}$; mono-Q fraction, $1.8 \mu \mathrm{g}$. Molecular mass markers were the same as those in panel A.

of full-length Rep52 was confirmed by immunoblotting with Rep-specific polyclonal and monoclonal antibodies $(13,15)$.

The Rep78 baculovirus construct contained AAV nucleotides 321 to 4493 . Oligonucleotide directed mutagenesis (19) was used to delete sequences between the left-end $X b a$ I site and the start codon for Rep translation in pIM29 to create plasmid 29dl1-320 (28). The $X b a$ I fragment from this plasmid was ligated into the $B a m \mathrm{HI}$ site of the baculovirus vector pVL941 (23) via XbaI linkers to make the transfer plasmid 941Xp5M (Fig. 2). Recombinant baculovirus was generated as described above except that recombinants containing Rep78 sequences were isolated by probing dot blots of end point dilutions of virus with oligonucleotide 5'-ATCTCGTAAAAC CCCGGCAT, which is specific for the region encoding the $\mathrm{NH}_{2}$ terminus of Rep78. The recombinant virus was subsequently plaque purified.

The Rep68 baculovirus construct was the same as the Rep78 clone except that the intron (AAV nucleotides 1906 to 2228) had been precisely deleted by oligonucleotide directed mutagenesis with oligonucleotide 5'-ACTACGCAGACAGATT GGCTCGAGGA. This deletion mutant was made in the background of pIM29, sequenced, and was designated $d$ lint $(19,26,27)$. Subsequently, the Bam HI fragment from 29dl1320 was inserted into the $B a m \mathrm{HI}$ site of $d$ lint to form plasmid $d l 1-320$ int. The $X b a \mathrm{I}$ fragment from $d l 1-320$ int was then inserted into the Bam HI site of the baculovirus transfer vector pVL941 to make the transfer plasmid 941XR68 (Fig. 2). Recombinant baculovirus was screened by dot blotting at limiting dilution with an AAV probe and then for the production of Rep68 by immunoblotting with the anti-52/40 monoclonal antibody or an anti-Rep peptide polyclonal antibody previously described $(13,15)$. In the case of the anti-52/40 mouse monoclonal antibody, an ammonium sulfate-precipitated fraction of an ascites preparation was used at a 1/10,000 dilution. All baculovirus plaques were replaqued three times before use.

Preparation of baculovirus extracts. One-molar salt extracts of baculovirus-infected nuclei were prepared as follows. One liter of SF9 suspension cells was grown to a density of $1.2 \times$ $10^{6}$ cells per ml in Graces media supplemented with $10 \%$ fetal calf serum, penicillin, streptomycin, and $0.1 \%$ pluronic S68
(GIBCO). The cells were infected with the appropriate baculovirus recombinant at a multiplicity of 8 and incubated at room temperature for 2 to 5 days. After harvesting by centrifugation, the cells were washed once by suspension in buffer $\mathrm{A}$ (20 mM HEPES [ $N$-2-hydroxyethylpiperazine- $N$ '-2-ethanesulfonic acid] [pH 7.5], $5 \mathrm{mM} \mathrm{KCl}, 0.5 \mathrm{mM} \mathrm{MgCl}_{2}$, and $0.5 \mathrm{mM}$ DTT) containing $10 \%$ sucrose. The cells were then suspended in $40 \mathrm{ml}$ of buffer $\mathrm{A}$ for $30 \mathrm{~min}$ on ice and lysed by 10 strokes with a type A Dounce homogenizer. Nuclei were harvested by centrifugation for $10 \mathrm{~min}$ at $2,000 \times g$ and resuspended in $8 \mathrm{ml}$ of buffer B (50 mM HEPES [pH 7.5], 10\% sucrose, $0.4 \mathrm{mM}$ EDTA, $0.3 \mathrm{mM}$ phenylmethylsulfonyl fluoride, $2 \mu \mathrm{g}$ of leupeptin per $\mathrm{ml}$, and $1 \mu \mathrm{g}$ of pepstatin per $\mathrm{ml}$ ). The suspension was adjusted to $1 \mathrm{M} \mathrm{NaCl}$, incubated on ice for $1 \mathrm{~h}$, and then centrifuged for $1 \mathrm{~h}$ at $100,000 \times g$. The protein concentration of the $1 \mathrm{M}$ nuclear S100 Rep extract was approximately 6 to 7 $\mathrm{mg} / \mathrm{ml}$ in a final volume of approximately $10 \mathrm{ml}$. For Rep78, the specific activity was approximately $2 \times 10^{4} \mathrm{U}$ (see below for definition) of replication activity per $\mathrm{mg}$ of protein.

Purification of baculovirus-expressed Rep68. Rep68 was purified to apparent homogeneity from a $1 \mathrm{M}$ nuclear extract of baculovirus-infected cells ( 1 liter) by a modification of the method previously described for Rep68 expressed in HeLa cells (15). Phenylsepharose and single-stranded DNA cellulose chromatography was performed as previously described (15). The single-stranded DNA cellulose pool was loaded onto a 1-ml HR5/5 mono-S column, washed with 10 column volumes of buffer C (25 mM Tris-HCl [pH 7.5], $0.1 \mathrm{mM}$ EDTA, $1 \mathrm{mM}$ DTT, $50 \mathrm{mM} \mathrm{NaCl}$ ) containing $0.1 \mathrm{mM}$ phenylmethylsulfonyl fluoride, $0.2 \mathrm{mM}$ pepstatin $\mathrm{A}$, and $15 \%$ glycerol. The column was washed with 10 column volumes of the starting buffer and eluted with 20 column volumes of a convex $50 \mathrm{mM}$-to- $1 \mathrm{M}$ $\mathrm{NaCl}$ gradient in buffer $\mathrm{C}$. Rep-containing fractions were identified by immunoblotting, pooled, and loaded onto a 1-ml HR5/5 mono-Q column. Elution from the mono-Q column was done in the same way as that from the mono-S column. The peak Rep-containing fraction was dialyzed against buffer $\mathrm{C}$ containing $50 \%$ glycerol, frozen in liquid nitrogen, and stored at $-80^{\circ} \mathrm{C}$.

Preparation of uninfected and Ad-infected HeLa cell extracts. Two kinds of extracts were used in this study. The first 
was a cytoplasmic $\mathrm{S} 100$ extract prepared as described previously by Stillman and Gluzman (44). The second was a cytoplasmic extract supplemented with a $0.2 \mathrm{M} \mathrm{NaCl}$ nuclear wash and was prepared essentially as described by Wobbe et al. (53) and modified by Ward and Berns (51). Both types of extracts are called S100 extracts in this article. HeLa suspension cells (11) were grown to a density of $5 \times 10^{5}$ cells per $\mathrm{ml}$ and harvested at $40 \mathrm{~h}$ postinfection by centrifugation to make uninfected extracts or extracts infected with Ad type 2 or type 5 ts 149 (obtained from Bruce Stillman, Cold Spring Harbor Laboratory) at a multiplicity of infection of 5 . After centrifugation at $100,000 \times g$ for $1 \mathrm{~h}$, the extracts were dialyzed overnight against $20 \mathrm{mM}$ Tris (pH 7.5)-50 mM NaCl-10\% glycerol-0.1 mM EDTA-1 mM DTT. The final protein concentrations of the uninfected and Ad-infected HeLa extracts were approximately $17 \mathrm{mg} / \mathrm{ml}$, and the extracts were stored at $-80^{\circ} \mathrm{C}$. Protein was measured with the Bradford reagent (Bio-Rad) using pooled bovine gamma globulin as the standard.

In vitro DNA replication assay. The standard AAV DNA replication assay was similar to the assay used previously for terminal resolution (42). It contained in $30 \mu 130 \mathrm{mM}$ HEPES (pH 7.5); $7 \mathrm{mM} \mathrm{MgCl} 2 ; 0.5 \mathrm{mM}$ DTT; $100 \mu \mathrm{M}$ each dATP, dGTP, dCTP, and dTTP; $25 \mu \mathrm{Ci}$ of $\left[\alpha{ }^{32} \mathrm{P}\right] \mathrm{dATP}(3 \mu \mathrm{Ci} / \mathrm{pmol})$; $4 \mathrm{mM}$ ATP; $40 \mathrm{mM}$ creatine phosphate; $1 \mu \mathrm{g}$ of creatine phosphokinase; $255 \mu \mathrm{g}$ of Ad-infected HeLa S100 extract; 0.1 $\mu \mathrm{g}$ of NE substrate DNA $(0.032$ pmol of AAV DNA or 300 pmol of nucleotide); and 1 to $80 \mathrm{U}$ of Rep78 or Rep68 baculovirus extract $(0.5$ to $8 \mu \mathrm{g})$. A unit of Rep activity was arbitrarily defined as an amount of Rep protein that would catalyze the incorporation of 1 pmol of radioactive deoxynucleoside monophosphate into DpnI-resistant monomer or dimer duplex AAV DNA in $2 \mathrm{~h}$ at $37^{\circ} \mathrm{C}$. Where indicated, the reactions were supplemented with CTP, GTP, and UTP $(0.2 \mathrm{mM}$ each). The reaction mixtures were incubated for a minimum of $2 \mathrm{~h}$ and up to $6 \mathrm{~h}$, as indicated. Following incubation, the reaction mixture was adjusted to $70 \mu \mathrm{l}$ containing $0.3 \%$ sodium dodecyl sulfate (SDS), $0.7 \mathrm{mg}$ of proteinase $\mathrm{K}$ per $\mathrm{ml}$, and $17 \mathrm{mM}$ EDTA. Proteinase $\mathrm{K}$ digestion was at $37^{\circ} \mathrm{C}$ for $1 \mathrm{~h}$. The products were then extracted with phenol and chloroform and precipitated with ethanol. The ethanol precipitate was dissolved in $18 \mu$ l of water and, where indicated, digested with $D p n \mathrm{I}$ for $2 \mathrm{~h}$ at $37^{\circ} \mathrm{C}$. The products (or a portion of them) were separated on either 0.8 or $1 \%$ agarose gels by electrophoresis for $4 \mathrm{~h}$ at $6 \mathrm{~V} / \mathrm{cm}$. The radioactivities in monomer and dimer RF products were counted in dried gels by a scanning gas flow (AMBIS) counter. X-ray film was exposed for $5 \mathrm{~min}$ to $16 \mathrm{~h}$ without a screen at room temperature.

In addition to the replication assay described above, total incorporation of radioactive precursor into acid-insoluble product was measured by the DE-81 filter paper (Whatman) method. Aliquots of products were spotted onto $1-\mathrm{cm}^{2} \mathrm{DE}-81$ filters and immediately immersed in $0.5 \mathrm{M}$ dibasic potassium phosphate ( $\mathrm{pH} \mathrm{8.6).} \mathrm{After} 3 \mathrm{~min}$, the filters were washed four more times ( $3 \mathrm{~min}$ each) with $0.5 \mathrm{M}$ phosphate solution, three times with water, and twice with ethanol. The filters were then dried and counted in a liquid scintillation counter.

\section{RESULTS}

Expression of Rep proteins in baculovirus vectors. In our previous experiments, we had purified Rep68 to apparent homogeneity and we had partially purified Rep78. Both were purified from HeLa cell extracts that had been infected with Ad and AAV and both were capable of resolving the AAV terminal repeats. However, neither of these proteins was able to promote net DNA synthesis, and although it was possible to separate the two proteins chromatographically, the yields were poor $(15,16,42)$. There were several possible explanations for the failure to achieve net synthesis. The simplest was that the level of Rep protein in HeLa cell crude or partially purified extracts was too low to support DNA synthesis. To obtain extracts that contained higher levels of Rep protein expression, we cloned Rep78, Rep68, and Rep52 into baculovirus vectors and prepared $1 \mathrm{M} \mathrm{NaCl}$ extracts of infected insect cell nuclei as described in Materials and Methods. Rep78 and Rep52 clones were constructed by inserting the appropriate region of the Rep coding sequence downstream of the baculovirus polyhedron promoter. The Rep68 clone was constructed in a similar way except that a cDNA clone was constructed by oligonucleotide mutagenesis to remove the Rep68 intron. Figure 2A illustrates the structure of the baculovirus clones and demonstrates that each of the clones expressed a Rep protein of the appropriate size by immunoblot analysis with a monoclonal antibody (13) which recognizes an epitope that is present in all four Rep proteins. In addition, all of the baculovirus clones synthesized Rep proteins that comigrated with Rep proteins synthesized in AAV-infected HeLa cells (data not shown). None of the Rep baculovirus clones produced detectable levels of the other Rep species (Fig. 2A). This confirmed the earlier report by Owens et al. (32) that none of the other Rep proteins could be detected in extracts from their independently isolated Rep78-expressing baculovirus clone. Two different preparations of Rep78 are shown in Fig. 2A. The earlier one (far left lane) contains a number of shorter Rep species due to proteolysis, but none of these migrate at the position of Rep68 or Rep52. The addition of a several protease inhibitors during extraction (see Materials and Methods) largely eliminated these breakdown products (Fig. 2A, far right lane). Rep78 and Rep68 are each found in HeLa cell extracts as two slightly differently migrating forms $(14,15,32,34)$. In contrast, and as reported previously by Owens et al. (32) for Rep78, the baculovirus-expressed Rep78 and -68 were each present as predominantly one species (Fig. 2A).

The crude $1 \mathrm{M}$ nuclear extracts in Fig. 2A were used as the source of the Rep proteins in the experiments described below. In the case of Rep68, we also purified the protein by a modification of a procedure we reported previously for the purification of Rep68 from HeLa cells (15) (Fig. 2B; see Materials and Methods). The most highly purified Rep68 fraction, a mono-Q fraction, is also shown in Fig. 2A (Rep68 $\mathrm{Q})$. This fraction was apparently homogeneous. When $1.8 \mu \mathrm{g}$ of the mono-Q fraction was analyzed on a silver-stained SDS-polyacrylamide gel (Fig. 2B), very little, if any, contamination by other protein species was detected. The Rep68 mono-Q fraction had a specific activity in the DNA replication assay of approximately $10^{6} \mathrm{U} / \mathrm{mg}$ of protein and was approximately 100 - to 200 -fold purified relative to the crude baculovirus preparation. The total yield of pure Rep68 was approximately 200 to $300 \mu \mathrm{g}$ from 1 liter of infected SF9 cells.

The ability of Rep78, -68 , and -52 to promote in vitro AAV DNA replication. To determine whether the baculovirus-expressed Rep proteins could promote AAV DNA synthesis, we tested each of them in an assay that was similar to the one we used previously for in vitro terminal resolution (42). The substrate for the reaction was NE DNA (Fig. 1), which consists of a linear AAV DNA molecule in which both ends are covalently joined (42). In addition to radiolabeled deoxynucleoside triphosphates, $\mathrm{MgCl}_{2}$, and an ATP-generating system, the reaction mixture contained one of the three Rep proteins (supplied in a crude baculovirus extract) and a crude 


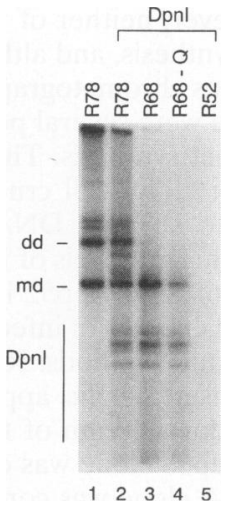

FIG. 3. Comparison of in vitro DNA synthesis by baculovirusexpressed Rep78, -68, and -52. Crude nuclear extracts of baculovirusexpressed Rep78, Rep68, and Rep52 and a purified preparation (mono-Q fraction) of baculovirus Rep68 (R68-Q) were incubated in the standard replication assay, except that it was adjusted to a total volume of $15 \mu \mathrm{l}$ containing $127 \mu \mathrm{g}$ of Ad-infected HeLa S100 extract and the samples were incubated at $37^{\circ} \mathrm{C}$ for $4 \mathrm{~h}$. Each Rep extract was titrated independently (not shown), and approximately the same number of replication units was added for each reaction: Rep78, 3.4 $\mu \mathrm{g}$; Rep68, $2.2 \mu \mathrm{g}$; R68-Q, $0.02 \mu \mathrm{g}$; Rep52, $3 \mu \mathrm{g}$. In the case of Rep52, DNA synthesis was seen at none of the Rep52 concentrations that were tried (not shown). The reaction mixtures were treated with proteinase $\mathrm{K}$ and one-sixth of each was analyzed on a $0.8 \%$ agarose gel either before (lane 1) or after (lanes 2 to 5) digestion with DpnI. dd and md, dimer duplex and monomer duplex AAV DNAs, respectively, as determined from $\lambda$ BstEII and BssHII molecular weight markers (not shown). The $D p n I$ restriction fragments are also indicated.

Ad-infected HeLa cell extract, which could supply the cellular or Ad-encoded replication factors that might be needed for DNA synthesis. The reaction mixtures were incubated for 2 to $6 \mathrm{~h}$. The products were then digested with $D p n \mathrm{I}$ and separated by electrophoresis on neutral agarose gels. Successful net synthesis would result in the production of DpnI-resistant monomer and dimer linear AAV RFs (Fig. 1).

We anticipated from previous genetic and biochemical studies $(4,16,40)$ that Rep52 would be unable to promote DNA synthesis by itself but that either Rep78 or Rep68 (or both) might be capable of generating DpnI-resistant AAV RFs. The products of a typical replication assay are shown in Fig. 3. As expected, Rep52 did not promote the incorporation of detectable levels of radioactive nucleotide into the NE substrate (Fig. 3, lane 5). The Rep52 reaction also served as an internal control which showed that, without the appropriate Rep protein, the enzymes in the HeLa cell extract or in the baculovirus extract were not capable by themselves of promoting significant background incorporation.

In contrast, the addition of either Rep68 or Rep78 produced DpnI-resistant AAV RF molecules (Fig. 3). In the case of Rep78, both monomer duplex and dimer duplex forms were synthesized (Fig. 3, lane 2) and the pattern of RFs was similar to that seen in vivo. Surprisingly, in the case of Rep68, only the monomer RF accumulated to any significant extent (Fig. 3, lane 3). To determine whether there might be an inhibitor in the Rep68 extracts that specifically prevented the accumulation of dimer RFs, we assayed the baculovirus-expressed Rep68 mono-Q fraction described above. It also produced primarily monomer RF (Fig. 3, lane 4). Thus, the inability to accumulate dimer RF species appeared to be an intrinsic property of Rep68, and it was the first clear difference that we have noticed between the biochemical activities of Rep68 and
Rep78. In addition, the experiments in which we used purified Rep68 to drive the replication reaction allowed us to calculate the ratio of Rep to DNA molecules in our assay. Titration of the mono-Q fraction (data not shown) revealed that optimal activity was achieved at a ratio of approximately 20 Rep68 molecules to 1 terminal repeat in the starting substrate. This ratio was twice that used in the experiment whose results are showed in Fig. 3 and was based on a theoretical molecular mass of Rep68 of $61,000 \mathrm{Da}$. We could not be certain, however, that all of the Rep68 molecules in the mono-Q fraction were active.

In addition to the monomer and dimer RF species generated by Rep78, several additional DpnI-resistant species were often seen, particularly a doublet that migrated just more slowly than the dimer duplex (Fig. 3, lanes 1 and 2). The larger species of this doublet had a mobility consistent with that of a tetramer RF molecule (see also Fig. 4B), but the structures of the other species in the doublet as well as the even higher-molecularweight forms in the Rep78 lanes have not yet been determined. All of these species have also been seen in vivo. Finally, a significant amount of radioactivity was incorporated into the DpnI-sensitive product during the course of the reaction. A comparison of the Rep52 lane with the Rep78 and Rep68 lanes (Fig. 3) suggests that a substantial amount of the DpnIsensitive incorporation was also Rep dependent. A possible explanation for this effect is that the DpnI-sensitive products represent partially replicated molecules that have incorporated radioactive nucleotides in a Rep-dependent fashion.

Reaction kinetics. As expected, the amount of radioactive nucleotide incorporated and the level of DpnI-resistant monomer and dimer RFs synthesized were dependent on the amount of Rep extract added to the reaction mixture (Fig. 4A). Although some incorporation into $D p n I$-sensitive products was seen at all levels of Rep, incorporation into the DpnI-resistant product appeared to be linear with Rep extract only after a threshold level of Rep extract had been reached (Fig. 4A). This may reflect the need to assemble a complex of Rep or Rep and cellular factors prior to full-length DNA synthesis. A time course of the DNA replication reaction also suggested the need to preassemble an active replication complex. No DpnIresistant full-length DNA was detected until approximately 30 min after the reaction was started (Fig. 4B). If total incorporation into DNA was measured by the DE-81 filter assay (see Materials and Methods), the lag was 17 to $20 \mathrm{~min}$ (data not shown). (The difference in lag time between the two assays presumably reflected the time that it took to synthesize the first full-length [DpnI-resistant] product after incorporation began.) After the initial 30-min lag, however, the accumulation of monomer and dimer RFs was approximately linear for the next $4 \mathrm{~h}$. This suggested that there was some rate-limiting step early in the reaction, perhaps the need to assemble a protein complex or the need to inactivate an inhibitor. The rate of incorporation of radioactive dAMP into monomer and dimer duplexes was approximately 20 to $40 \mathrm{pmol} / \mathrm{h}$ after the initial $30-\mathrm{min}$ lag. This is similar to the rate of incorporation in the SV40 DNA replication systems reported previously (21, 44, 53). Given the amount of starting substrate DNA in the standard $30-\mu$ l reaction mixture $(300$ pmol of nucleotide; approximately $75 \mathrm{pmol}$ of dAMP), the incorporation of 150 pmol of dAMP in approximately $4 \mathrm{~h}$ (Fig. 4B) is consistent with each starting molecule being replicated up to two times.

Semiconservative replication. To confirm that the DpnIresistant DNA produced was the result of semiconservative replication, a reaction was performed without any radioactive label and with bromo-dUTP in place of dTTP. The products of the reaction were separated in a $\mathrm{CsCl}$ density gradient, and the species in each fraction were analyzed by Southern hybridiza- 

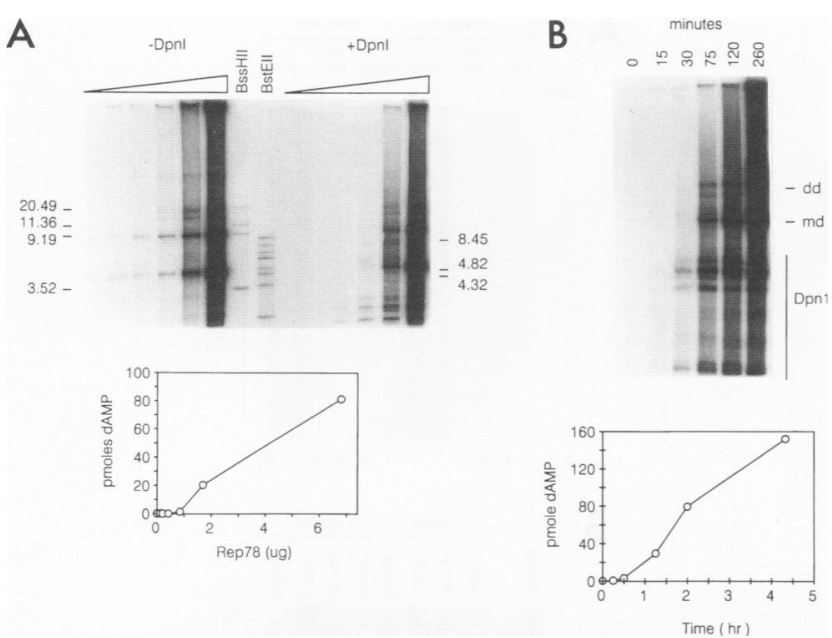

FIG. 4. (A) Titration of Rep78. The standard DNA replication assay was used, except that it was adjusted to a total volume of $15 \mu \mathrm{l}$ containing $127 \mu \mathrm{g}$ of Ad-infected HeLa S100 extract and (from left to right) $0.1,0.2,0.4,0.9,1.7$, or $6.8 \mu \mathrm{g}$ of Rep78 baculovirus nuclear extract. The reaction mixtures were incubated for $4 \mathrm{~h}$ and treated with proteinase K. Aliquots (one-fifth) of each reaction were electrophoresed on a $1 \%$ agarose gel both before (left) and after (right) DpnI digestion. The BssHII and BstEII lanes contain $\lambda$ bacteriophage DNA digested with the indicated restriction enzyme and $3^{\prime}$ labeled with Klenow fragment to provide size markers. The sizes of the BssHII fragments are shown on the left. The relevant BstEII fragment sizes are indicated on the right. Monomer length sub201 AAV DNA in which both ends have been resolved is expected to be $4.702 \mathrm{kbp}$ long $(36,43)$; the dimer RF with both ends resolved is expected to be 9.279 $\mathrm{kbp}$. Each unresolved end would reduce the size of an RF molecule by $0.124 \mathrm{kbp}$. The amount of $\left[{ }^{32} \mathrm{P}\right] \mathrm{dAMP}$ incorporated into $D p n \mathrm{I}$-resistant monomer and dimer RF molecules was counted and is displayed graphically below. The incorporation was adjusted to represent the amount incorporated in a $15-\mu \mathrm{l}$ reaction mixture. (B) Time course of AAV DNA synthesis. The standard AAV DNA replication reaction mixture $(30 \mu \mathrm{l})$ contained $3.4 \mu \mathrm{g}$ of crude Rep78 nuclear extract and $255 \mu \mathrm{g}$ of Ad-infected HeLa S100 extract. The reaction mixtures were incubated for the indicated time, treated with proteinase $\mathrm{K}$ and $D p n \mathrm{I}$, and electrophoresed on a $1 \%$ agarose gel. The positions of the monomer (md) and dimer (dd) duplex RF species are indicated on the right, as are the incompletely replicated $D p n I$-sensitive products (Dpn1). The amount of $\left.{ }^{32} \mathrm{P}\right] \mathrm{dAMP}$ incorporated into DpnI-resistant monomer and dimer RF molecules was counted and is displayed graphically below.

tion after electrophoresis on a neutral agarose gel (Fig. 5). Virtually all of the starting material was shifted from the light to the hybrid density. This suggested that nearly all of the starting substrate had been used during the course of the 6-h reaction and had undergone at least one round of replication. The small amount of starting substrate that remained at the light density was monomer length, as expected. In contrast, hybrid density fractions contained both monomer and dimer species, as might be expected of material that had undergone DNA replication. In addition, a small fraction of the products $(<5 \%)$ were found at the heavy position of the gradient, suggesting that some of the product DNA was the result of at least two rounds of full-length DNA replication.

Orientation of the terminal sequences in product DNA. As mentioned earlier, the terminal repeat sequences are inverted during each round of terminal resolution. Actively replicating AAV DNA contains equal amounts of the two terminal repeat orientations, flip and flop $(24,25,35)$. The starting NE substrate was constructed so that both ends would generate the

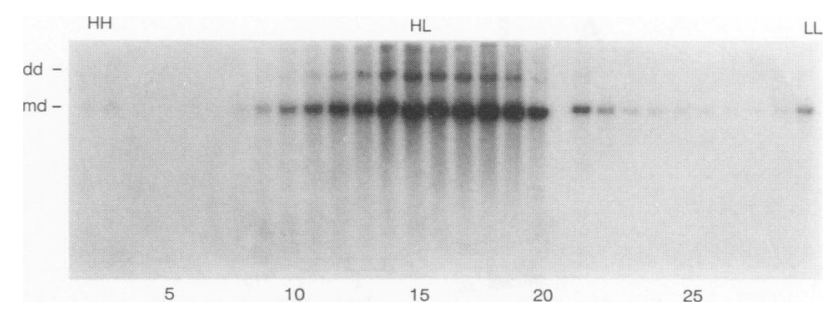

FIG. 5. Isopycnic centrifugation of in vitro-synthesized AAV DNA. DNA was synthesized under standard reaction conditions except that $100 \mu \mathrm{M} 5$ ' bromo-dUTP was used instead of dTTP in a total reaction volume of $120 \mu \mathrm{l}$ for $6 \mathrm{~h}$ in the absence of radioactive deoxynucleoside triphosphates. After incubation, the reaction products were treated with proteinase $\mathrm{K}$ and centrifuged to equilibrium in a neutral $\mathrm{CsCl}$ solution containing $10 \mathrm{mM}$ Tris- $\mathrm{HCl}$ (pH 7.5), $1 \mathrm{mM}$ EDTA, and 1.750 $\mathrm{g}$ of $\mathrm{CsCl}$ per $\mathrm{ml}$ in a total volume of $4 \mathrm{ml}$. Fractions were collected from the bottom and electrophoresed on a $0.8 \%$ agarose gel. The presence of AAV DNA was detected by Southern blotting using the randomly labeled AAV-containing PvuII fragment from psub201 plasmid DNA as a probe. The density of the fractions was determined by weight to confirm that the density gradient was linear (not shown). The expected positions of unreplicated, light-light (LL) DNA, once replicated heavy-light (HL) DNA, and doubly replicated heavy-heavy (HH) DNA are indicated at the top. md, monomer duplex; dd, dimer duplex.

flop orientation during the first round of resolution $(36,42)$. Reinitiation and strand displacement synthesis of the fulllength molecule would then generate a covalently closed end that would generate the flip orientation after a second round of resolution. In our previous experiments, in which no net synthesis and only one round of terminal resolution had occurred, we detected only the flop orientation in the products of the reaction (42). Under the conditions of net synthesis used here, we expected to find both orientations in the products of the reaction. To see whether we could detect both orientations of the terminal repeats, we isolated the resolved terminal $\mathrm{XbaI}$ fragment from the products of the replication reaction as described previously (42) (see the legends to Fig. 6A and 7B). Digestion of the terminal $X b a$ I fragment with $S m a I$ revealed that both orientations were present in the products of the reaction in approximately equal amounts (Fig. 6A). This suggested that, indeed, more than one round of terminal resolution was occurring during the course of the replication reaction.

In addition, we demonstrated in our previous work that the apparent molecular weight of the in vitro-resolved SmaI terminal flop fragment was slightly larger than expected (42). This was due to the fact that, during in vitro resolution, a molecule of Rep protein was covalently attached to the $5^{\prime}$ end of the terminal repeat, and even after treatment of the products with proteinase $\mathrm{K}$, a portion of the Rep protein still remained attached $(15,40,42)$. When the SmaI flop fragment was treated with pronase and proteinase $K$, its apparent molecular weight was reduced further, presumably because additional amino acids of the Rep protein were removed. To see whether the same would be true of the terminal fragments generated in the DNA replication assay, some of the SmaI terminal fragments were digested with pronase in addition to proteinase $\mathrm{K}$ (Fig. 6A). Both the flip (68 bp) and the flop (46 bp) terminal fragments were shifted to a lower molecular weight after digestion with pronase. This suggested that the bulk of the product DNA generated in the in vitro replication reaction contained a covalently attached Rep protein.

Requirement for a functional AAV origin. To determine 

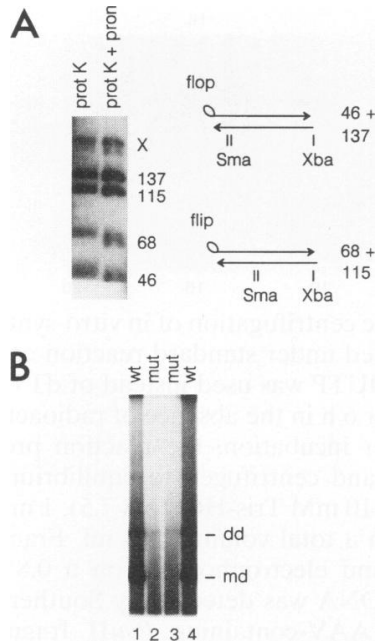

FIG. 6. (A) Orientation of the resolved termini. The standard DNA replication reaction mixture was adjusted to $15 \mu$ l containing 127 $\mu \mathrm{g}$ of Ad-infected HeLa S100 extract and $1.7 \mu \mathrm{g}$ of $1 \mathrm{M}$ baculovirus Rep78 nuclear extract and incubated at $37^{\circ} \mathrm{C}$ for $4.5 \mathrm{~h}$. The products were treated with proteinase $\mathrm{K}$, digested with $\mathrm{XbaI}$ and PstI, and electrophoresed on a $6 \%$ polyacrylamide gel. The resolved, ${ }^{32} \mathrm{P}$-labeled $X b a I$ terminal fragment was isolated from the gel, digested with SmaI, and electrophoresed on an $8 \%$ nondenaturing polyacrylamide gel. The left lane contains products that had been treated only with proteinase $\mathrm{K}$ (prot $\mathrm{K}$ ); the right lane contains fragments that had been further treated with pronase (pron) prior to electrophoresis. The diagram on the right illustrates the relative positions of $S m a I$ sites in the flip and flop orientations of the resolved termini. Arrowheads indicate the $3^{\prime}$ ends of each strand. Ovals indicate the fact that, after proteolytic digestion, a portion of the Rep protein remains covalently attached to the $5^{\prime}$ end of the terminal repeat. Numbers indicate the sizes (in base pairs) of the expected SmaI-digested products. (The small 10-bp fragment that contains the sequence between the two SmaI sites is lost on these gels.) As shown previously by Snyder et al. (42), the variation in the sizes of the flip-terminal SmaI fragment (68 bp) and the flop-terminal fragment (46 bp) reflects the fact that the alternate proteolytic treatments leave different amounts of the Rep protein covalently attached to the fragments. X, the resolved terminal $X b a \mathrm{I}$ fragments that escaped SmaI digestion or were partially digested (immediately below the $\mathrm{X}$ fragment). (B) Comparison of wild-type and mutant origins. Duplicate reactions were performed with wild-type (wt) or HpaIA mutant (mut) NE template DNA. The mutant contains an 8-bp linker inserted at the trs site. It is 50 -fold reduced in its ability to be nicked by the Rep protein in vitro (41) and defective for DNA replication in vivo (56a). The DNA replication reaction conditions were the same as those for Fig. 4B. The products of the reaction were electrophoresed on a $0.8 \%$ agarose gel.

whether the in vitro AAV replication reaction was specific for a wild-type AAV origin, a mutant origin was tested in the reaction. The HpaIA mutant substrate consisted of NE DNA in which both termini had identical 8-bp linker insertions near the terminal resolution site. In previous work, we have shown that this mutant substrate is approximately 50 -fold less efficient in its ability to be cut by the Rep protein at the terminal resolution site in vitro (41), and Samulski and his colleagues (34a) have shown that it is equally defective for DNA replication in vivo. When the HpaIA mutant substrate was compared with the wild-type origin in the in vitro replication assay, there was a clear preference for the wild-type terminal repeat (Fig. $6 \mathrm{~B})$. Only small amounts of monomer and dimer RF species could be detected in the products of the reaction with the mutant substrate, even after prolonged exposure of the gel.

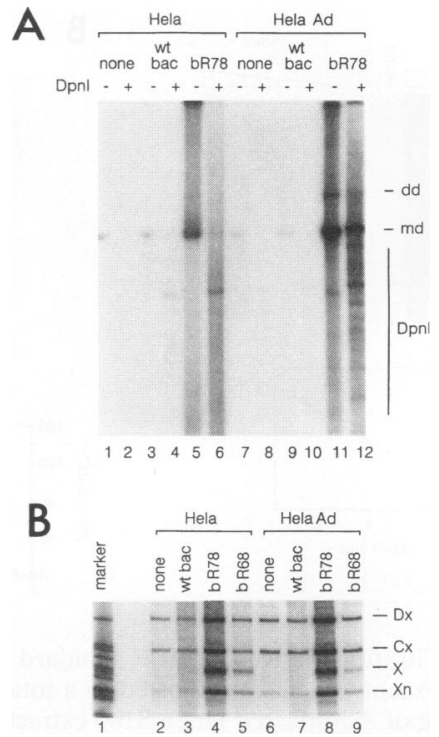

FIG. 7. Requirement for Rep protein and an Ad-infected extract. (A) NE DNA was incubated with S100 extracts from either uninfected or Ad-infected HeLa cells in the presence of no additional extract, a wild-type baculovirus (wt bac) nuclear extract, or a baculovirus nuclear extract containing Rep78 (bR78) under standard replication reaction conditions in a total reaction volume of $120 \mu \mathrm{l}$ for $6 \mathrm{~h}$ at $37^{\circ} \mathrm{C}$. Wild-type and Rep78 baculovirus extracts $(20.4 \mu \mathrm{g})$ were added to the indicated reactions mixtures. Following incubation, the reaction mixtures were treated with proteinase $\mathrm{K}$, and one-sixth each reaction mixture was analyzed on a $0.8 \%$ agarose gel, either with $(+)$ or without (-) DpnI digestion. dd and md, DpnI-resistant dimer duplex and monomer duplex AAV DNAs, respectively; DpnI, the DpnI-sensitive partially replicated products. (B) Aliquots of the reaction mixtures in panel $\mathrm{A}$ and two additional reaction mixtures with baculovirus Rep68 extract were treated with $P s t \mathrm{I}$ and $\mathrm{XbaI}$ and electrophoresed on a 6\% polyacrylamide gel. $\mathrm{Cx}$ and Dx, internal Pst $\mathrm{I}-\mathrm{XbaI}$ fragments; $\mathrm{X}$ and $\mathrm{Xn}$, resolved and unresolved terminal $X b a \mathrm{I}$ fragments, respectively. The marker lane contains 3'-labeled pBR322 MspI fragments.

Requirement for an Ad-infected extract. As mentioned earlier, AAV DNA replication is unusual in that coinfection with Ad is usually necessary to establish fully permissive conditions for DNA replication. To see whether the in vitro reaction also depended on Ad infection, the abilities of uninfected and Ad-infected HeLa cell extracts to support AAV DNA replication were compared (Fig. 7 and 8). The results in Fig. 7A show that, in the presence of Rep78, uninfected HeLa extracts supported incorporation of radioactive nucleotides into product DNA but virtually all of the product DNA was DpnI sensitive. In contrast, the Ad-infected extract efficiently generated DpnI-resistant monomer and dimer RF species. As expected, both the uninfected and the Ad-infected extracts promoted little incorporation in the absence of Rep protein or in the presence of a wild-type baculovirus extract. The difference between uninfected and Ad-infected HeLa extracts was confirmed by titrating each extract in the presence of a constant amount of Rep (Fig. 8). As more Ad-infected extract was added to the reaction mixture, the yield of DpnI-resistant monomer and dimer RF products increased (Fig. 8, lanes 5 to 8 ). In contrast, it was difficult to detect $D p n I$-resistant products with the uninfected HeLa extract at any concentration that was tried (Fig. 8, lanes 1 to 4). The same difference between uninfected and Ad-infected extracts was seen when Rep68 was used in the replication assay (Fig. 8, lanes 9 and 10). The effect 


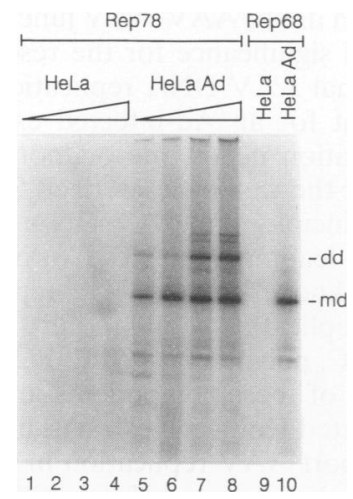

FIG. 8. Titration of uninfected and Ad-infected HeLa cell cytoplasmic extracts. DNA was synthesized in a standard reaction mixture that had been adjusted to $15 \mu \mathrm{l}$, containing $3.4 \mu \mathrm{g}$ of Rep78 (lanes 1 to 8 ) or $0.9 \mu \mathrm{g}$ of Rep68 (lanes 9 and 10) baculovirus nuclear extract (1 M) and increasing amounts of an uninfected HeLa S100 extract (lanes 1 to 4 and 9) or an Ad-infected HeLa extract (lanes 5 to 8 and 10). Extract amounts (in micrograms): 76.5 (lanes 1 and 5), 102 (lanes 2 and 6), 127 (lanes $3,7,9$, and 10), and 153 (lanes 4 and 8 ). The reaction mixtures were incubated at $37^{\circ} \mathrm{C}$ for $4 \mathrm{~h}$, treated with proteinase $\mathrm{K}$, and digested with $D p n I$. One-sixth of each reaction was separated on a $0.8 \%$ agarose gel. dd and md, DpnI-resistant dimer duplex and monomer duplex AAV DNAs, respectively.

of Ad infection suggested that some factor(s) that directly participated in AAV DNA replication was missing (or reduced) in an uninfected HeLa extract. Other explanations, however, are also possible (see Discussion).

Finally, in previous work we had demonstrated that an uninfected HeLa cell extract was capable of supporting the terminal resolution reaction (Fig. 1) provided it was supplemented with purified Rep protein (40). To see whether this was true of the HeLa extracts used in this study, we digested the products of the in vitro replication assay with $X b a \mathbf{I}$ and PstI and compared the yields of the resolved terminal $X b a I$ fragment produced in the presence of uninfected and Ad-infected extracts (Fig. 7B). Both types of extracts were found to generate approximately the same level of the resolved $\mathrm{XbaI}$ fragment in the presence of either Rep78 or Rep68 (Fig. 7B; compare the $\mathrm{X}$ fragments in lanes 4 and 8 with those in lanes 5 and 9). This confirmed our earlier results and suggested that the block in AAV DNA replication with an uninfected HeLa extract occurred at some step after terminal resolution, either in the formation of the reinitiation intermediate or in elongation (Fig. 1).

Other components required for the reaction. As already discussed, the in vitro reaction required the NE DNA substrate with wild-type terminal repeats and either Rep78 or Rep68 (Table 1). As expected from other DNA replication systems, the AAV replication reaction also required the presence of $\mathrm{MgCl}_{2}, \mathrm{ATP}$, and an ATP-generating system (creatine phosphate and creatine phosphokinase). Omission of the other ribonucleoside triphosphates (UTP, GTP, and CTP) did not affect the reaction (Table 1 ).

\section{DISCUSSION}

We have developed an efficient in vitro AAV DNA replication assay that should be useful for identifying and purifying the cellular (or helper virus) components involved in AAV DNA replication. Our assay is up to 100 -fold more efficient than previously described assays, both in the utilization of the
TABLE 1. Components required for in vitro replication of $\mathrm{AAV} \mathrm{DNA}^{a}$

\begin{tabular}{|c|c|}
\hline Component omitted & $\begin{array}{l}\% \text { of complete } \\
\text { reaction }\end{array}$ \\
\hline None.. & 100 \\
\hline $\mathrm{MgCl}_{2}$ & $<0.5$ \\
\hline ATP & 3.3 \\
\hline CTP, GTP, UTP & 100 \\
\hline dGTP, dCTP, dTTP .... & $<0.5$ \\
\hline $\mathrm{CP}, \mathrm{CPK}^{b} \ldots \ldots$ & $<0.5$ \\
\hline NE substrate & $<0.5$ \\
\hline Rep78 ................... & $<0.5$ \\
\hline+ Baculovirus $^{c}$. & $<0.5$ \\
\hline Ad-infected HeLa S100... & $<0.5$ \\
\hline $\mathrm{S} 100^{d}$ & $<0.5$ \\
\hline
\end{tabular}

${ }^{a}$ See "In vitro DNA replication assay" for the complete reaction mixture. ${ }^{b} \mathrm{CP}$ and $\mathrm{CPK}$, creatine phosphate and creatine phosphate kinase, respectively.

${ }^{c}$ A wild type-infected baculovirus extract was substituted for the Rep78containing baculovirus extract.

${ }^{d}$ An uninfected HeLa S100 extract was substituted for the Ad-infected S100 extract.

starting substrate and in the rate of incorporation $(12,59)$. Density shift experiments indicated that almost all of the starting substrate was replicated at least once and a portion of the substrate (less than 5\%) was replicated twice during a 6-h incubation (Fig. 5). The level of labeled nucleotide incorporated suggested that two rounds of DNA replication could occur (Fig. 4). The discrepancy between these experiments was likely due to an uncertainty in the amount of starting substrate used or to inherent differences in the extracts used in the two experiments. A comparison of the DNA replication assay presented here and that of Hong et al. (12) suggests that the difference in efficiency is probably due to the use of circular AAV plasmids as the starting substrate for replication. Before AAV replication can proceed efficiently, a linear molecule must be rescued from the circular plasmid. Aside from being a potential rate-limiting step, we (6-8) and Ward and Berns (51) have shown that in vitro rescue of AAV linear DNA can occur by at least two different mechanisms which do not always produce products that are active substrates for subsequent AAV DNA replication (31a). It should also be noted that our results are not necessarily in conflict with those of the experiments reported by Yalkinoglu et al. (59). We have suggested previously that there may be two different mechanisms for AAV DNA replication, the one described here and a second one which may be used during integration of AAV proviruses (29). The latter may rely on the use of the AAV terminal repeat as a cellular origin, as suggested by Yalkinoglu et al. (59).

The reaction faithfully reflected in vivo AAV DNA replication by several criteria. First, the major replicative intermediates formed during the reaction were monomer and dimer duplex linear DNAs, and most of the products were DpnI resistant, suggesting that full-length AAV DNA replication had occurred. Second, the reaction required the presence of one of the two larger Rep proteins, Rep78 or Rep68. Third, a mutant origin that we had previously shown to be defective for nicking by Rep68 (41) was also defective as a substrate in the DNA replication assay. Fourth, the in vitro products contained both orientations of the terminal repeat (flip and flop) in approximately equal amounts, as in vivo. This indicated that at least two rounds of terminal resolution had occurred in a large fraction of the termini. Fifth, the reaction was much more efficient in the presence of an Ad-infected HeLa cell extract 
than it was with an uninfected cell extract. This was also consistent with AAV replication in vivo.

The stoichiometry of the products that we observed in the in vitro reaction can be explained by the current model for AAV DNA replication as follows. The hairpinned termini in the starting NE substrate are first resolved at both ends to produce the flop orientation at both ends. One of the resolved ends is then converted to the double hairpin intermediate (illustrated in Fig. 1 as the reinitiation substrate), which then primes a round of full-length strand displacement synthesis to produce a duplex molecule with one hairpinned (or covalently closed) end and a single-stranded molecule. Resolution of the hairpinned end in the duplex molecule would then produce a duplex (heavy-light) molecule that is resolved at both ends and has one flip end and one flop end. Similarly, the singlestranded product of the strand displacement reaction would be elongated by hairpin priming from the $3^{\prime}$ end to produce a duplex product with one end in the hairpinned configuration. Terminal resolution of this molecule would also produce a double-stranded product with one flip end and one flop end. Thus, the net result of one round of strand displacement synthesis, followed by elongation of the single-stranded product and the resolution of all hairpinned ends, would be two double-stranded hybrid (heavy-light) molecules and an equal molar ratio of flip and flop ends. This is consistent with our observations (Fig. 5 and 6A). As yet, we have not examined the product DNA to determine the distribution of flip and flop ends on individual molecules. Dimer molecules (Fig. 1) would be the result of reinitiation and strand displacement synthesis on molecules in which only one of the ends had been resolved. Such events, followed by the resolution of all dimer termini, would also produce hybrid (heavy-light) molecules with equimolar ratios of flip and flop ends. This also is consistent with our observations (Fig. 5). Finally, we note that other mechanisms could explain the equimolar ratio of flip and flop ends in the product DNA. For example, repeated resolution and partial elongation could occur at an end to generate what would appear to be an equimolar flip/flop ratio. Such a mechanism would be expected to generate small DNA products that contained only the terminal repeat sequence. Although such products were not seen during the course of these studies, we have not rigorously excluded them.

One aspect of the reaction that may not faithfully reflect in vivo AAV DNA replication was the covalent linkage of the Rep protein to the ends of product DNA. Although we have not demonstrated this conclusively, the shift in size in the terminal SmaI fragments after sequential proteolytic digestion (Fig. 6) suggested that most of the product synthesized in vitro contained a covalently attached Rep protein. It is not known whether this is also the case with replicative intermediates in vivo, but there is no evidence that AAV DNA packaged in vivo contains a covalently attached protein. Thus, some processing step that would be required to remove the covalently attached protein prior to or during packaging does not occur in our in vitro DNA replication reaction. It should be noted that no AAV capsid proteins were present in our reactions and Rep and capsid proteins have been found to colocalize in AAVinfected nuclei (13).

In previous work, we have compared the biochemical properties of Rep78 and Rep68 with respect to DNA binding, trs endonuclease activity, and DNA helicase activity (14-16). The inability of Rep68 to generate significant levels of dimer RF intermediates is the first qualitative difference that we have found between the two enzymes. The reason for the difference is not yet clear, but one possible explanation is that Rep68 is more efficient in resolving internal AAV terminal sequences that are present in a dimer AAV-AAV junction. This may have some physiological significance for the rescue of AAV proviruses and for normal AAV DNA replication.

The requirement for an Ad-infected extract suggests that AAV DNA replication needs one or more components that are not needed for the well characterized SV40 DNA replication system. In principle, the AAV DNA replication mechanism involves only leading-strand DNA synthesis. Extrapolating from what is known about SV40 replication, we might expect that AAV replication would require (in addition to Rep protein) RPA, RFC, polymerase $\delta$, and PCNA (20, 21, 30, 33, $49,50,52-56)$. All of these components are present in ample amounts in uninfected HeLa cell extracts, but these extracts do not efficiently support AAV replication in vitro. This suggests that some additional component that is either coded by Ad or coded by the cell and induced following Ad infection is required for AAV DNA replication. Previous genetic experiments have shown that neither the Ad DNA polymerase nor the Ad terminal protein is required for AAV DNA replication or virus production $(17,29 a, 45)$. Studies of Ad DNA-binding protein mutants suggest that, although they do affect virus production, they have little, if any, effect on AAV DNA replication in vivo ( 3 ; also references therein). Thus, the missing component required for AAV DNA synthesis is likely to be a cellular enzyme. Finally, both Rep78 and Rep68 were capable of resolving the hairpinned terminal sequences in the presence of uninfected cell extracts (40) (Fig. 7). Thus, whatever the missing component is, it appears to be required either for the reinitiation step of AAV DNA replication (in which a putative double hairpinned intermediate is formed) or for elongation during strand displacement synthesis (Fig. 1). It remains to be seen whether the difference between uninfected and Ad-infected extracts we observed is due to a novel cellular factor or simply to the induction of higher levels of known replication factors by Ad infection. We note also that there are several other possible explanations for the improved DNA synthesis in Ad-infected extracts. For example, uninfected extracts may contain an inhibitor of AAV DNA replication that is inactivated during Ad infections. Alternatively, Ad infection may lead to posttranslational modifications of some of the known cellular replication proteins. Ultimately, it will be necessary to reconstitute AAV DNA replication with purified proteins to determine the role of the helper virus.

\section{ACKNOWLEDGMENTS}

This work was supported by a program project grant (N.M.) from the National Cancer Institute (5 PO1 CA2814607), by a grant (RO1 GM3572302) from the National Institute of General Medical Sciences (N.M.), by a Public Health Service training grant (AI25530) from the National Institutes of Health (D.M.M.), and by an American Cancer Society fellowship (N.M.). Some of this work was done in the laboratory of Bruce Stillman at Cold Spring Harbor Laboratories while N.M. was on sabbatical leave and was supported by a grant (CA13106) to Bruce Stillman from the National Cancer Institute.

We thank Bruce Stillman and the members of his laboratory for helpful discussions.

\section{REFERENCES}

1. Berns, K. I. 1990. Parvoviridae and their replication, p. 1743-1763. In B. N. Fields and D. M. Knipe (ed.), Virology, 2nd ed., vol. 2. Raven Press, New York.

2. Berns, K. I., and W. W. Hauswirth. 1979. Adeno-associated viruses. Adv. Virus Res. 25:407-449.

3. Carter, B. J., B. A. Antoni, and D. F. Klessig. 1992. Adenovirus containing a deletion of the early region $2 \mathrm{~A}$ gene allows growth of adeno-associated virus with decreased efficiency. Virology 191: 473-476. 
4. Chejanovsky, N., and B. J. Carter. 1989. Mutagenesis of an AUG codon in the adeno-associated virus rep gene. Virology 173:120 128.

5. Fairman, M. P., and B. Stillman. 1988. Cellular factors required for multiple stages of SV40 replication in vitro. EMBO J. 7:12111218.

6. Gottlieb, J., and N. Muzyczka. 1988. In vitro excision of adenoassociated virus DNA from recombinant plasmids: isolation of an enzyme fraction from HeLa cells that cleaves DNA at poly(G) sequences. Mol. Cell. Biol. 6:2513-2522.

7. Gottlieb, J., and N. Muzyczka. 1990. Purification and characterization of HeLa endonuclease R. J. Biol. Chem. 265:10836-10841.

8. Gottlieb, J., and N. Muzyczka. 1990. Substrate specificity of HeLa endonuclease R. J. Biol. Chem. 265:10842-10850.

9. Hauswirth, W. W., and K. I. Berns. 1977. Origin and termination of adeno-associated virus DNA replication. Virology 78:488-499.

10. Hauswirth, W. W., and K. I. Berns. 1979. Adeno-associated virus DNA replication: non unit length molecules. Virology 93:57-68.

11. Hermonat, P. L., M. A. Labow, R. Wright, K. I. Berns, and N. Muzyczka. 1984. Genetics of adeno-associated virus: isolation and preliminary characterization of adeno-associated virus type 2 mutants. J. Virol. 51:329-333.

12. Hong, G., P. Ward, and K. I. Berns. 1992. In vitro replication of adeno-associated virus DNA. Proc. Natl. Acad. Sci. USA 89:46734677.

13. Hunter, L. A., and R. J. Samulski. 1991. Colocalization of adenoassociated virus Rep and capsid proteins in the nuclei of infected cells. J. Virol. 66:317-324.

14. Im, D.-S., and N. Muzyczka. 1989. Factors that bind to adenoassociated virus terminal repeats. J. Virol. 63:3095-3104.

15. Im, D.-S., and N. Muzyczka. 1990. The AAV origin binding protein Rep68 is an ATP-dependent site-specific endonuclease with DNA helicase activity. Cell 61:447-457.

16. Im, D.-S., and N. Muzyczka. 1992. Partial purification of adenoassociated virus Rep78, Rep68, Rep52, and Rep40 and their biochemical characterization. J. Virol. 66:1119-1128.

17. Janik, J. E., M. M. Huston, and J. A. Rose. 1981. Locations of adenovirus genes required for the replication of adeno-associated virus. Proc. Natl. Acad. Sci. USA 78:1925-1929.

18. Kornberg, A., and T. A. Baker. 1991. DNA replication, 2nd ed. W. H. Freeman, New York.

19. Kunkel, T. A. 1985. Rapid and efficient site-specific mutagenesis without phenotypic selection. Proc. Natl. Acad. Sci. USA 82:488492.

20. Lee, S. H., T. Eki, and J. Hruwitz. 1989. Synthesis of DNA containing the simian virus 40 origin of replication by the combined action of DNA polymerases alpha and delta. Proc. Natl. Acad. Sci. USA 86:7361-7365.

21. Li, J. J., and T. J. Kelly. 1984. Simian virus 40 DNA replication in vitro. Proc. Natl. Acad. Sci. USA 81:6973-6977.

22. Luckow, V. A., and M. D. Summers. 1988. Trends in the development of baculovirus expression vectors. Bio/Technology 6:47-55.

23. Luckow, V. A., and M. D. Summers. 1989. High level expression of nonfused genes with Autographa californica nuclear polyhedrosis virus expression vectors. Virology 170:31-39.

24. Lusby, E., R. Bohenzky, and K. I. Berns. 1981. Inverted terminal repetitions in adeno-associated virus DNA: independence of the orientation at either end of the genome. J. Virol. 37:1083-1086.

25. Lusby, E., K. H. Fife, and K. I. Berns. 1980. Nucleotide sequence of the inverted terminal repetition in adeno-associated virus DNA. J. Virol. 34:402-409.

26. Maniatis, T., E. F. Fritsch, and J. Sambrook. 1982. Molecular cloning: a laboratory manual. Cold Spring Harbor Laboratory, Cold Spring Harbor, N.Y.

27. Maxam, A. M., and W. Gilbert. 1980. Sequencing end-labeled DNA with base-specific cleavages. Methods Enzymol. 65:499-560.

28. McCarty, D. M., M. Christensen, and N. Muzyczka. 1991. Sequences required for coordinate induction of adeno-associated virus p19 and p40 promoters by Rep protein. J. Virol. 65:2936 2945.

29. McLaughlin, S. K., P. Collis, P. L. Hermonat, and N. Muzyczka. 1988. Adeno-associated virus general transduction vectors: analysis of proviral structures. J. Virol. 62:1963-1973.
29a.McLaughlin, S. K., and N. Muzyczka. Unpublished data.

30. Melendy, T., and B. Stillman. 1991. Purification of DNA polymerase $\delta$ as an essential simian virus 40 DNA replication factor. J. Biol. Chem. 266:1942-1949.

31. Muzyczka, N. 1992. Use of adeno-associated virus as a general transduction vector for mammalian cells. Curr. Top. Microbiol. Immunol. 158:98-129.

31a.Ni, T.-H., and N. Muzyczka. Unpublished data.

32. Owens, R. A., J. P. Trempe, N. Chejanovsky, and B. J. Carter. 1991. Adeno-associated virus Rep proteins produced in insect and mammalian expression systems: wild-type and dominant-negative mutant proteins bind to the viral replication origin. Virology 184:14-21.

33. Prelich, G., and B. Stillman. 1988. Coordinated leading and lagging strand synthesis during SV40 DNA replication in vitro requires PCNA. Cell 53:117-126.

34. Redemann, B. E., E. Mendelson, and B. J. Carter. 1989. Adenoassociated virus Rep protein synthesis during productive infection. J. Virol. 63:873-882.

34a.Samulski, R. J., et al. Unpublished data.

35. Samulski, R. J., K. I. Berns, M. Tan, and N. Muzyczka. 1982. Cloning of adeno-associated virus into pBR322: rescue of intact virus from the recombinant plasmid in human cells. Proc. Natl. Acad. Sci. USA 79:2077-2081.

36. Samulski, R. J., L.-S. Chang, and T. Shenk. 1987. A recombinant plasmid from which an infectious adeno-associated virus genome can be excised in vitro and its use to study viral replication. J. Virol. 61:3096-3101.

37. Samulski, R. J., A. Srivastava, K. I. Berns, and N. Muzyczka. 1983. Rescue of adeno-associated virus from recombinant plasmids: gene correction within the terminal repeats of AAV. Cell 33:135143.

38. Schlehofer, J. R., M. Ehrbar, and H. zur Hausen. 1986. Vaccinia virus, herpes simplex virus, and carcinogens induce DNA amplification in a human cell line and support replication of a helper virus dependent parvovirus. Virology 152:110-117.

39. Senepathy, P., J. Tratschin, and B. J. Carter. 1984. Replication of adeno-associated virus DNA. Complementation of naturally occurring rep ${ }^{-}$mutants by a wild-type genome or an ori ${ }^{-}$mutant and correction of terminal deletions. J. Mol. Biol. 179:1-20.

40. Snyder, R. O., D.-S. Im, and N. Muzyczka. 1990. Evidence for covalent attachment of the adeno-associated virus (AAV) Rep protein to the ends of the AAV genome. J. Virol. 64:6204-6213.

41. Snyder, R. O., D.-S. Im, T. Ni, X. Xiao, R. J. Samulski, and N. Muzyczka. 1993. Features of the adeno-associated virus origin involved in substrate recognition by the viral Rep protein. J. Virol. 67:6096-6104.

42. Snyder, R. O., R. J. Samulski, and N. Muzyczka. 1990. In vitro resolution of covalently joined AAV chromosome ends. Cell 60:105-113.

43. Srivastava, A., E. W. Lusby, and K. I. Berns. 1983. Nucleotide sequence and organization of the adeno-associated virus 2 genome. J. Virol. 45:555-564.

44. Stillman, B. W., and Y. Gluzman. 1985. Replication and supercoiling of simian virus 40 DNA in cell extracts from human cells. Moll. Cell. Biol. 5:2051-2060.

45. Straus, S. E., H. S. Ginsburg, and J. A. Rose. 1976. DNA-minus temperature-sensitive mutants of adenovirus type 5 help adenovirus-associated virus replication. J. Virol. 17:140-148.

46. Straus, S. E., E. D. Sebring, and J. A. Rose. 1976. Concatemers of alternating plus and minus strands are intermediates in adenovirus-associated virus DNA synthesis. Proc. Natl. Acad. Sci. USA 73:742-746.

47. Tattersall, P., and D. C. Ward. 1976. Rolling hairpin model for replication of parvovirus and linear chromosomal DNA. Nature (London) 263:106-109.

48. Tratschin, J., I. L. Miller, and B. J. Carter. 1984. Genetic analysis of adeno-associated virus: properties of deletion mutants constructed in vitro and evidence for an adeno-associated virus replication function. J. Virol. 51:611-619.

49. Tsurimoto, T., and B. Stillman. 1991. Replication factors required for SV40 DNA replication in vitro. I. DNA structure-specific recognition of a primer-template junction by eukaryotic DNA 
polymerases and their accessory proteins. J. Biol. Chem. 266:1950 1960.

50. Tsurimoto, T., and B. Stillman. 1991. Replication factors required for SV40 DNA replication in vitro. II. Switching of DNA polymerase $\alpha$ and $\delta$ during initiation of leading and lagging strand synthesis. J. Biol. Chem. 266:1961-1968.

51. Ward, P., and K. I. Berns. 1991. In vitro rescue of an integrated hybrid adeno-associated virus/simian virus 40 genome. J. Mol. Biol. 218:791-804.

52. Weinberg, D. H., and T. J. Kelly. 1989. Requirement for two DNA polymerases for the synthesis of SV40 DNA in vitro. Proc. Natl. Acad. Sci. USA 86:9742-9746.

53. Wobbe, C. R., F. Dean, L. Weissbach, and J. Hurwitz. 1985. In vitro replication of duplex circular DNA containing the simian virus 40 DNA origin site. Proc. Natl. Acad. Sci. USA 82:57105714 .

54. Wobbe, C. R., L. Weissbach, J. A. Borowiec, F. B. Dean, Y. Murakami, P. Bullock, and J. Hurwitz. 1987. Replication of simian virus 40 origin containing DNA in vitro with purified proteins. Proc. Natl. Acad. Sci. USA 84:1834-1838.
55. Wold, M. S., and T. J. Kelly. 1988. Purification and characterization of replication protein $\mathrm{A}$, a cellular protein required for in vitro replication of simian virus 40 DNA. Proc. Natl. Acad. Sci. USA 83:2523-2527.

56. Wold, M. S., J. J. Li, and T. J. Kelly. 1987. Initiation of simian virus 40 DNA replication: large tumor antigen and origin dependent unwinding of the template. Proc. Natl. Acad. Sci. USA 84:36433647.

56a.Xiao, X., and R. J. Samulski. Unpublished observations.

57. Yacobson, B., T. Koch, and E. Winocour. 1987. Replication of adeno-associated virus in synchronized cells without the addition of a helper virus. J. Virol. 61:972-981.

58. Yalkinoglu, A. O., R. Heilbronn, A. Burkle, J. R. Schlehofer, and H. zur Hausen. 1988. DNA amplification of adeno-associated virus as a response to cellular genotoxic stress. Cancer Res. 48:31233129.

59. Yalkinoglu, A. O., H. Zentgraf, and U. Hubscher. 1991. Origin of adeno-associated virus DNA replication is a target of carcinogeninducible DNA replication. J. Virol. 65:3175-3184. 